\title{
Wnt signaling and polarity in freshwater sponges
}

\author{
Pamela J. Windsor Reid ${ }^{1,2^{*}}$, Eugueni Matveev' ${ }^{1}$, Alexandra McClymont ${ }^{1}$, Dora Posfai ${ }^{3,4}$, April L. Hill ${ }^{3}$ \\ and Sally P. Leys ${ }^{1 *}$
}

\begin{abstract}
Background: The Wnt signaling pathway is uniquely metazoan and used in many processes during development, including the formation of polarity and body axes. In sponges, one of the earliest diverging animal groups, Wnt pathway genes have diverse expression patterns in different groups including along the anterior-posterior axis of two sponge larvae, and in the osculum and ostia of others. We studied the function of Wnt signaling and body polarity formation through expression, knockdown, and larval manipulation in several freshwater sponge species.

Results: Sponge Wnts fall into sponge-specific and sponge-class specific subfamilies of Wht proteins. Notably Wnt genes were not found in transcriptomes of the glass sponge Aphrocallistes vastus. Wnt and its signaling genes were expressed in archaeocytes of the mesohyl throughout developing freshwater sponges. Osculum formation was enhanced by GSK3 knockdown, and Wnt antagonists inhibited both osculum development and regeneration. Using dye tracking we found that the posterior poles of freshwater sponge larvae give rise to tissue that will form the osculum following metamorphosis.

Conclusions: Together the data indicate that while components of canonical Wnt signaling may be used in development and maintenance of osculum tissue, it is likely that Wnt signaling itself occurs between individual cells rather than whole tissues or structures in freshwater sponges.
\end{abstract}

Keywords: Wnt, $\beta$-catenin, Porifera, Body polarity, Metazoan evolution, Osculum

\section{Background}

One of the most intriguing transitions in evolution is that of the origin of the first multicellular animals. We now know that transition involved a massive expansion of gene families (e.g. [1-3]), and while the ancestry of many developmental pathways can be traced to premetazoan eukaryotes (e.g. [4-6]), a select few, such as the Wnt pathway are uniquely metazoan.

Wnts are one of the most widely used ligands in the development of many animal phyla [7], but one of their central roles is that of axial patterning. Canonical Wnt/ $\beta$-catenin ( $\beta$-cat) signaling allows $\beta$-catenin to enter the nucleus to bind TCF-LEF and activate downstream genes. Evidence for the canonical Wnt/ $\beta$-cat signaling pathway in cnidarians include polarized expression patterns of Wnt and Wnt pathway components [8], experiments to

\footnotetext{
* Correspondence: reidp22@macewan.ca; sleys@ualberta.ca ${ }^{1}$ Department of Biological Sciences, University of Alberta, Edmonton, AB, Canada

Full list of author information is available at the end of the article
}

activate Wnt using glycogen synthase kinase-3 (GSK-3) inhibitors such as lithium chloride, alsterpaullone or BIO [9-12], and localization of $\beta$-catenin within the nuclei of developing embryos [10]. Classical transplantation experiments have also shown that the blastoporal region in the cnidarian Nematostella can induce a second axis, indicating that in both cnidarians and vertebrates canonical Wnt signaling establishes body axes [13].

The pre-history of the Wnt pathway is intriguing. GSK-3 is known from the choanoflagellate Monosiga brevicollis [6], and the slime mold Dictyostelium has GSK-3, which interacts with a $\beta$-catenin homolog Aardvark (Aar), as well as several homologs of the Frizzled (fz) receptor [14]. If the Wnt pathway in its entirety is unknown in pre-metazoans, when did canonical Wnt signaling arise and for which developmental functions?

As the earliest diverging groups, both ctenophores and sponges should shed light on these questions. The ctenophore Pleurobrachia pileus has four Wnt genes, all of which are expressed around the mouth [15]. Four 
Wnts were also found in Mnemiopsis leidyi but their expression is associated with the aboral pole and tentacle apparatus [16]. Pharmacological experiments to activate the Wnt pathway produced no effect in M. leidyi [16]; experiments have not been reported in P. pileus. So, despite the presence of core Wnt pathway components in ctenophores, it is as yet unclear whether canonical signaling functions to polarize the ctenophore body plan.

The four classes of sponges (Porifera) unsurprisingly have distinct larval development and adult body plans. The demosponge Amphimedon queenslandica has three Wnts, one of which (AquwntA) is expressed at the posterior pole of the larva $[17,18]$, but few other genes in the Wnt pathway show polarized expression in the larva. In the homoscleromorph Oscarella carmela eight Wnts have been found, and in O. lobularis only three, one of which shows expression around the ostium or incurrent openings for the water filtration system [19]. A calcareous sponge, Sycon ciliatum, has a surprising $21 \mathrm{Wnt}$ genes, many of which show polarized expression, but pharmacological experiments showed no effect on the expression patterns or polarity of the sponge [20]. Another demosponge, Halisarca dujardini, has a larger complement of Wnt genes (ten), and two of them are expressed at the posterior end of the larvae while others are expressed in the adult osculum and along the body axis. Their role in axial polarity however, remains unclear [21].

Two other studies have suggested a role for canonical Wnt signaling in formation of the sponge feeding canals. Treatments with GSK3 inhibitors caused ectopic oscula, the vent of the aquiferous system in the freshwater demosponge Ephydatia muelleri, [22] and more ostia water intake pores - to form in the homoscleromorph sponge Oscarella lobularis, where OlowntII is expressed [19]. In E. muelleri transplantation of the osculum induced canals to grow towards it, suggesting the osculum has organizer properties [22]. Together these data suggest that Wnt has some role in the establishment of overall body polarity in a sponge - either swimming polarity in the larva and/or the polarity of the aquiferous canal (feeding) system.

The evidence for canonical $\mathrm{Wnt} / \mathrm{\beta}$-cat signaling in sponges and ctenophores is therefore still equivocal. The apparent absence of components of the Wnt pathway in some sponges (e.g. axin in Aphrocallistes vastus and Sycon coactum and Wnt and dsh in A. vastus; [3]), the inability of pharmacological experiments to affect polarized development in Sycon [20], and the failure to pull down axin bound to $\beta$-catenin in Ephydatia muelleri [23] leave doubt, as do the different expression patterns and inability to activate the pathway using pharmacology in the ctenophore $M$. leidyi. It is possible that canonical
Wnt signaling is used in defining axial polarity only in eumetazoans (Cnidaria + Bilateria), and expression at poles of larvae and adult sponges is coincidental. In this scenario components of the Wnt pathway would have been used for other functions in unicellular eukaryotes and pre-eumetazoan animals. For example the genome of Dictyostelium contains several elements of Wnt/ $/$-catenin signaling $(F z, G S K 3 \beta$, a $\beta$-catenin homolog and a putative $d k k ;[11,14])$; it is hypothesized that the origin of Wnt and dsh in early metazoans was key in connecting the pieces of this pathway together [7]. Canonical Wnt signaling would have arisen for some function of coordinating grander cell movements, but not necessarily involved in determining the location of polarized structures in pre-eumetazoan animals. Here we examine this hypothesis using a diversity of approaches - in situ expression patterns, RNAi knockdowns, larval manipulations, and pharmacological inhibition experiments - in a diversity of freshwater sponge model systems.

\section{Results}

\section{Phylogenetic relationships of sponge Wnts}

We compiled wht genes from 17 species of sponge from all 4 Poriferan classes, either by searches of our own transcriptomes or of those available online (see Additional file 1), and on NCBI (http://www.ncbi.nlm.nih.gov). Wht genes are in all sponge classes with the notable exception of the glass sponge Aphrocallistes vastus. We identified up to $3 \mathrm{Wnts}$ in demosponges (Ephydatia muelleri, Spongilla lacustris, Eunapius fragilis, Ircinia fasciculata, Chondrilla nucula, Petrosia ficiformis, Pseudospongosorites suberitoides), 5 Wnts in the homoscleromorph Corticium candelabrum and 13 in the calcareous sponge Sycon coactum. Sponge Wnts had the typical 23-24 conserved cysteine residue positions along the protein and a semi-conserved RWNC motif towards the $\mathrm{N}$ terminal typical to the Wnt protein sequence (Additional file 2).

To determine the subfamily relationships within sponge Wnts we conducted maximum likelihood analyses of fulllength aligned sponge Wnt sequences as shown in the unrooted consensus tree in Fig. 1 (all raw trees are included in Additional file 3). We consistently recovered 3 subfamilies of demosponge Wnts with moderate to high support: PorWntA (95/93/100), PorWntB (98/100/100) and PorWntC (58/67/94). We also recovered the previously reported WntI (92/95/100) and WntII (100/100/ 100) subfamilies from homoscleromorph sponges, although some Wnt sequences from homoscleromorphs did not appear to group with other Wnts (CcaWntX1, CcaWntX2, OcaWnt2X, OcaWnt5X and OcaWnt6X). The transcriptome of $O$. carmela also has alternate transcripts of WntII, here named OcaWntIIa, b and c. We were able to assign some Wnts from Sycon coactum and Leucosolenia complicata to the families described in [20], which 


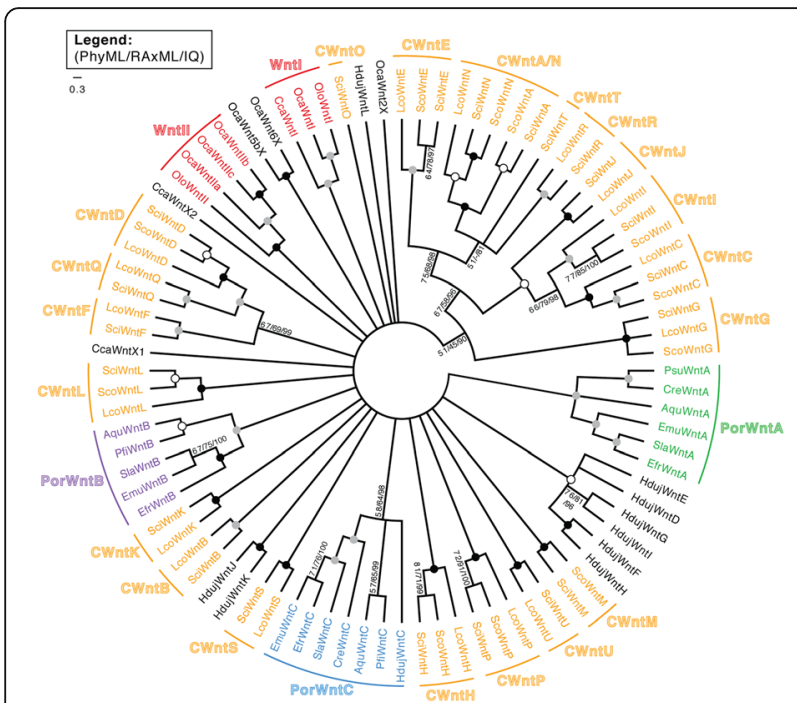

Fig. 1 Poriferan Wnt relationships. Consensus tree of Wnt relationships from sponges based on PhyML, RAXML and IQ-TREE analyses. Support values indicated are from PhyML/RAxML/IQ-TREE bootstrap support. A closed circle $(\bullet)$ bootstrap support of 100 for all analyses, a grey circle (๑) indicates support over 90 for all analyses, and an open circle (o) is support of over 80 for all analyses. Taxon abbreviations: Aqu, Amphimedon queenslandica; Ava, Aphrocallistes vastus; Cca, Corticium candelabrum; Cre, Crella elegans; Emu, Ephydatia muelleri; Efr, Eunapius fragilis; Hduj, Halisarca dujardini; Lco, Leucosolenia complicata, Oca, Oscarella carmela; Olo, Oscarella lobularis; Pfi, Petrosia ficiformis; Psu, Pseudospongosorites suberitoides; Sla, Spongilla lacustris; Sci, Sycon ciliatum; Sco, Sycon coactum

are here denoted as CWntA through U. These subfamilies were highly supported (98-100 in all analyses), except CWntA and N, which appear to form a highly-supported clade of paralogues $(100 / 100 / 100)$ with less support for the internal nodes (CWntA-86/88/100; CWntN-84/73/100). Support in the backbone and deeper nodes of the tree was consistently low, and thus much of the tree was collapsed into a polytomy (Fig. 1). The assignment of sponge Wnts to subfamilies of previously unpublished or unanalyzed sequences is given in Additional file 4.

Sponge Wnts do not fall into the Wnt subfamilies of other metazoans (Additional file 5). Vertebrate Wnt sequences fall with high support into distinct, well-defined families, however backbone support throughout the tree was weak and thus no conclusions can be made about the branching order of Wnt subfamilies and the relationships between them.

\section{Wnt pathway gene expression during development from the gemmule}

We examined the expression patterns of Wnt $/ \beta$-catenin pathway genes ( $3 w n t, 3 f z, 1 d s h, 1$ GSK3, $1 \beta$-cat, and 1 tcf/lef) in 2 and 5 days post hatch (dph) juveniles of $E$. muelleri using both colourimetric (NBT/BCIP) and fluorophore (FISH) based in situ hybridization detection methods.

Expression of all genes was detected in single cells in the mesohyl, either in the choanosome or in a region peripheral to the choanosome (Fig. 2a, b). Where the sponge tissues are thin at the periphery, archaeocytes can be seen crawling in the collagenous mesohyl (Fig. 2b, inset).

We used silicatein $M 2$, a gene expressed in sclerocytes in the growing sponge as previously described in [24] as a positive control. At 2 and $5 \mathrm{dph}$ expression of silicatein M2 was detected in sclerocytes, which could be identified by their elongate shape (Fig. 2c, d), but never in amoeboid cells at the periphery. In $5 \mathrm{dph}$ sponge, silicatein $M 2$ probe was also detected elsewhere, however it is unclear whether it is cellular or extracellular, or if these were cells fated to become sclerocytes (Fig. 2c, d). In contrast, wnt mRNAs were only detected in cells of 5 dph sponges that had a complete aquiferous system. In these sponges wntA, $B$, and $C$ mRNA was detected in amoeboid cells in the mesohyl at the periphery of the sponge and throughout the choanosome (Fig. 2e-g). Probe targeting $\beta$-cat was detected in similarly-shaped amoeboid cells along the periphery of $5 \mathrm{dph}$ sponges (Fig. 2h), however these cells lacked the thin filopodial-like projections seen in cells labelled by $w n t$ probes. In all cases wnts and $\beta$-catenin were detected in only a subset of amoeboid cells at the periphery. Double in situ hybridizations suggest that wnt $A$ and $C$ might be co-localized in cells but wntA and $B$ are not (Fig. 2i, j). We made a custom antibody to a fusion protein of a conserved region of sponge $\beta$-catenin (Additional file 6). Interestingly this custom antibody also labelled amoeboid cells in the mesohyl in Spongilla lacustus (Fig. 2k). The antibody labelled both the cytoplasm, regions around the cell membrane, as well as distinct filopodia. Images from Fig. $2 \mathrm{f}-\mathrm{k}$ are shown as separate channels in Additional file 7.

Probes targeting other Wnt pathway genes $(f z, d s h$, GSK-3, and tcf/lef) labelled cells in the mesohyl around the choanocyte chambers (Fig. 3a-f), but it was not possible to determine exactly what cells these were. The relationships between various cell types and regions is illustrated in Fig. 3g. We detected $w n t$ and $\beta$-cat mRNA in amoeboid cells of the periphery, and $f z, d s h$, GSK3 and tcf/ lef mRNAs were detected in cells within the choanosome.

Interestingly, we found that in all sponges with a fully developed aquiferous system ( $5 \mathrm{dph}$ ) every probe, including non-sponge controls such as Danio rerio hemoglobin and engrailed as well as sense probe controls, labelled a region adjacent to choanocyte chambers (Additional file 8). Because no probe negative controls showed no label, the non-specific label above cannot be due to a lack of blocking endogenous alkaline phosphatases or peroxidase activity (Additional file 8). 


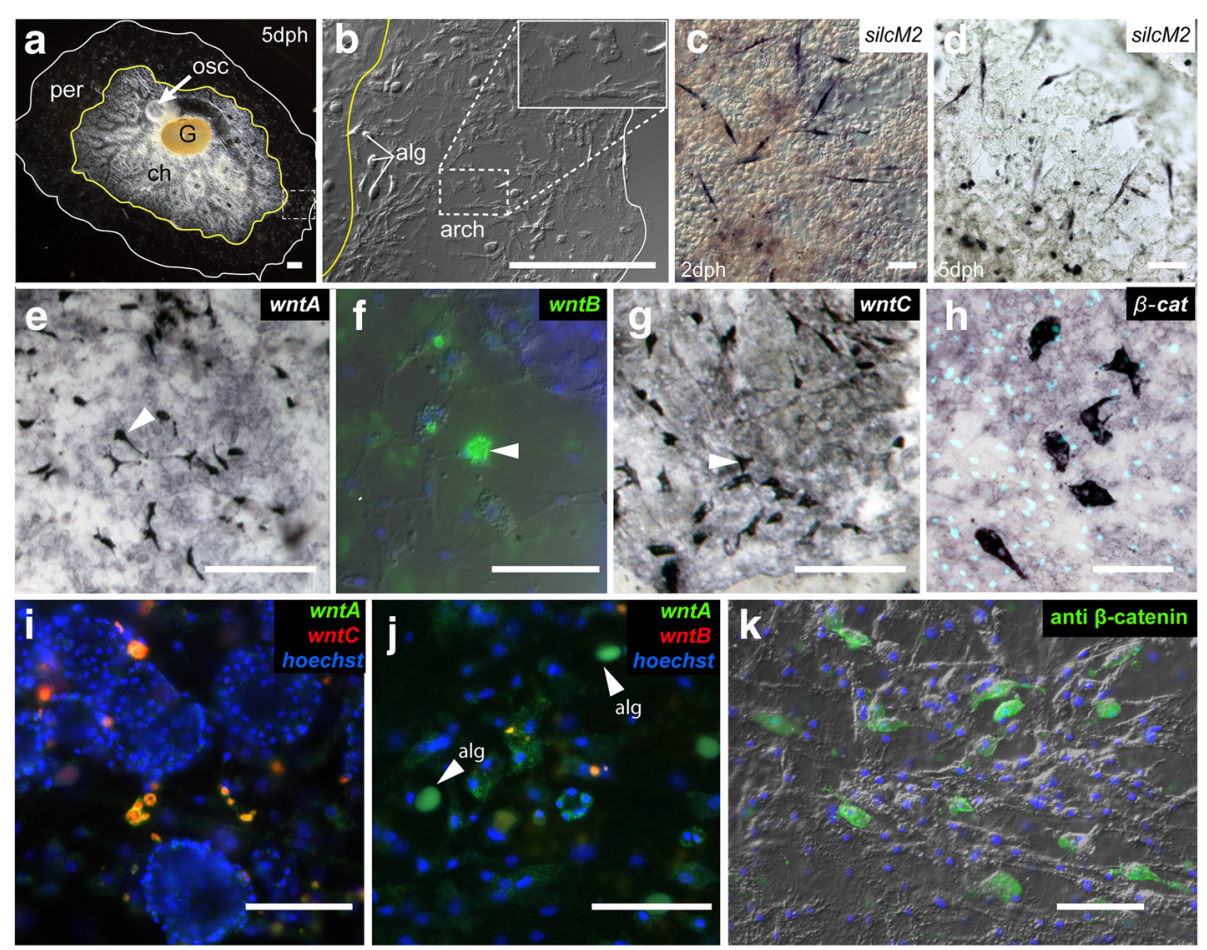

Fig. 2 Expression of wnts and $\beta$-catenin reveal cellular and not regional expression patterns. a) Whole sponge at 5 dph showing regions of the body including the gemmule, G, osculum, osc, choanosome, ch with choanocyte chambers and canals and bordered in yellow - and the peripheral region, per, shown by the white border. b) Expanded view of boxed area in a). Archaeocytes (arch) and algal cells (alg) in the peripheral region shown by DIC. Inset shows amoeboid archaeocytes. c), d) silicatein M2 (positive control) in situ hybridization in 2 and 5 dph sponges, respectively (whole mount top; inset bottom). Sclerocytes are spindle-like in shape (arrowheads). e) - h) Expression of EmuwntA, EmuwntB, EmuwntC, and Emuß-catenin mRNA in archaeocytes of the peripheral region. Double in situ hybridizations of $\mathbf{i}$ ) $w n t A$ and $C$ in the choanosome near the choanocyte chambers and $\mathbf{j})$ wnt $A$ and $B$ in the peripheral region. $\mathbf{c}$ ) - e) and $\mathbf{g}$ ) - h) NBT/BCIP, $\mathbf{f}$ ) Fluorescent in situ hybridizations with wnt labels in green and/or red and nuclei in blue (Hoechst). k) Anti- $\beta$-catenin staining (green) in peripheral tissue of Spongilla lacustris, nuclei are indicated in blue (Hoechst), and merged with DIC. Arrowheads in $\mathbf{e}$ ), $\mathbf{f}$ ) and $\mathbf{g}$ ) indicated labeled archaeocytes, arrowheads in $\mathbf{j}$ ) indicate algal symbiont auto-fluorescence (alg). Scales: $50 \mu \mathrm{m}$

\section{RNAi downregulation of GSK-3}

Given that inhibition of GSK-3 protein by lithium chloride and alsterpaullone was shown to result in the formation of ectopic oscula in E. muelleri [22], we tested whether knockdown of GSK-3 RNA by dsRNA would lead to similar changes in the aquiferous system (i.e., production of additional oscula). GSK-3 dsRNA-treated E. muelleri sponges often developed multiple (2-5) oscula, and canals radiated from the center of the sponge in an irregular branching pattern (Fig. 4a). Untreated sponges and sponges treated with control dsRNA (for silicatein M2, a gene involved in spicule formation) developed a normal canal system with bifurcating canals and typically only one osculum (Fig. 4b, c), although occasionally two oscula were observed. The osculum rises away from the sponge roughly $100 \mu \mathrm{m}$ or more and so diagrams are used to illustrate both the location of oscula and the branching pattern of canals attached to each (Fig. 4a-c).

As sponges hatched at different times, and because previous experiments had shown that extra oscula generated by lithium chloride treatment are eventually resorbed
[22], we counted oscula several times over the $48 \mathrm{~h}$ treatment and recorded the maximum number of oscula observed (Fig. 4d). Overall, the data showed significant differences in the number of oscula (Kruskal-Wallis test: test statistic $=19.430, \mathrm{df}=2, p<0.0005$; Chi-Square test: critical value $=23.73, x^{2}=9.488, \alpha=0.05, v=4, p<0.01$ ) and the maximum number of oscula observed in GSK-3 dsRNA treated sponges was significantly higher than in sponges untreated or in silicatein M2 dsRNA treated sponges (Dunn test: $n=85, p \leq 0.005, \mathrm{k}=3$ ). The number of oscula in untreated $(n=76)$ and silicatein M2 dsRNA treated $(n=33)$ sponges was not significantly different (Dunn test: $p>0.05$ ). A $28 \%$ knockdown of EmuGSK-3 for this experiment was confirmed with two internal replicates by qPCR, using EmuEf1- $\alpha$ as the housekeeping reference gene (Additional file 9). Though the RNAi technique as currently used in E. muelleri only results in knockdown of $\sim 30 \%$ (range of $\sim 20-50 \%$ depending on the gene tested), phenotypes of EmuGSK-3 knockdown sponges were distinct from those observed in other knockdown experiments targeting different genes [25-27]. 

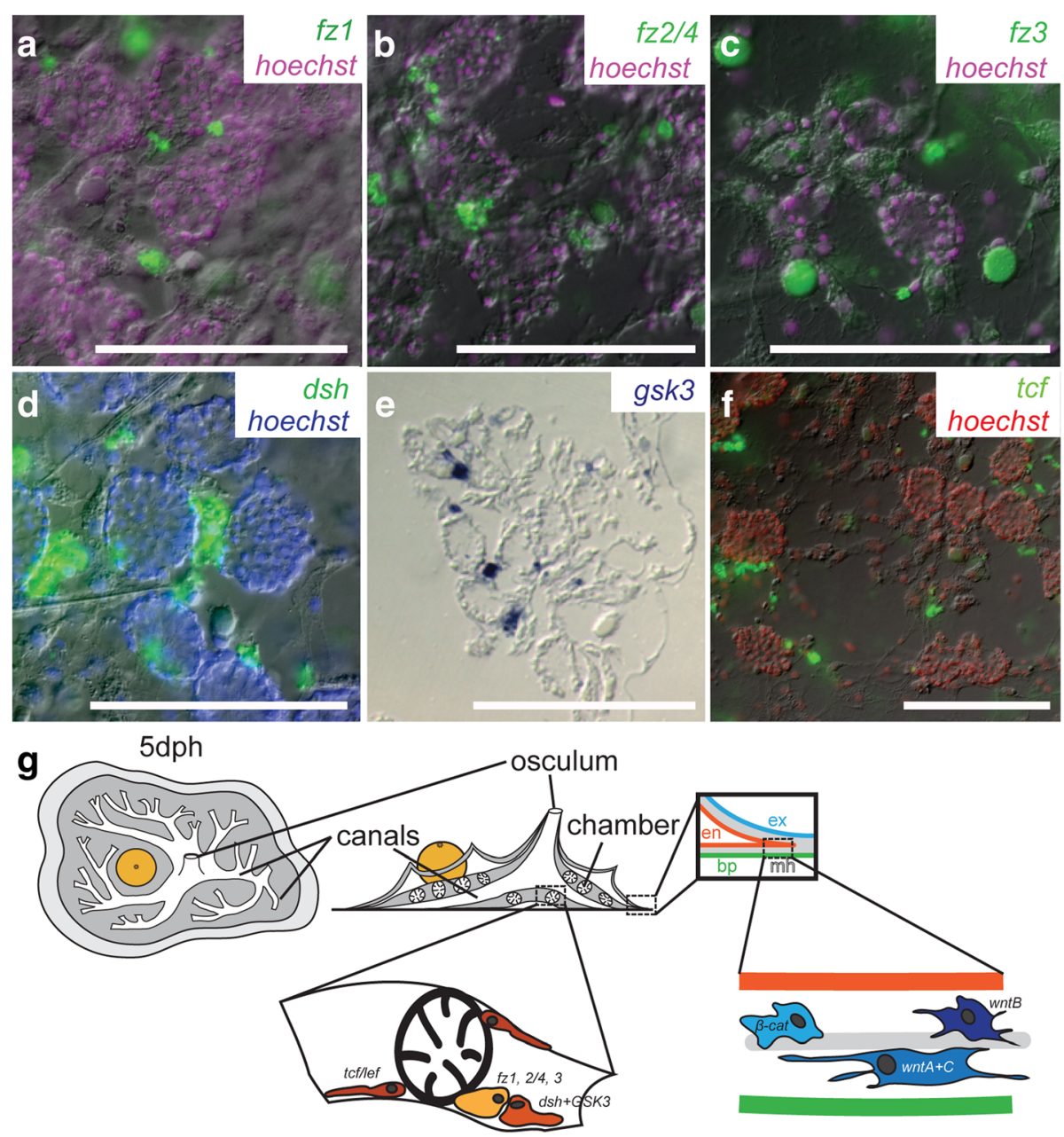

Fig. 3 Expression of other Wnt pathway genes: $f z 1, f z 2 / 4, f z 3, d s h, g s k 3, t c f$. a-d), ff $f z 1, f z 2 / 4, f z 3, d s h$ and tcf mRNA expression, respectively (green), from fluorescent in situ hybridization with nuclear counterstain (magenta, blue or red, Hoechst) near choanocyte chambers. e) $g s k 3$ expression in the same region, visualized with NBT/BCIP and sectioned in epoxy. g) Summary diagram of the sponge body plan (top view, left; side view, right) at $5 \mathrm{dph}$ with cell types and regions shown where Wnt pathway gene expression was detected. The small inset on the periphery shows layers of different cell types and linings of sponge regions: the exopinacoderm (ex, blue), the endopinacoderm (en, red) and basopinacoderm (bp, green), between these layers is the mesohyl (mh, gray) which contains many cell types including archaeocytes. In the choanosome (inset, right), we find expression of $f z, d s h, g s k 3$ and tcf. In the periphery (inset, left) we find whts and $\beta$-cat. Modified from [61]. Scales: $100 \mu m$
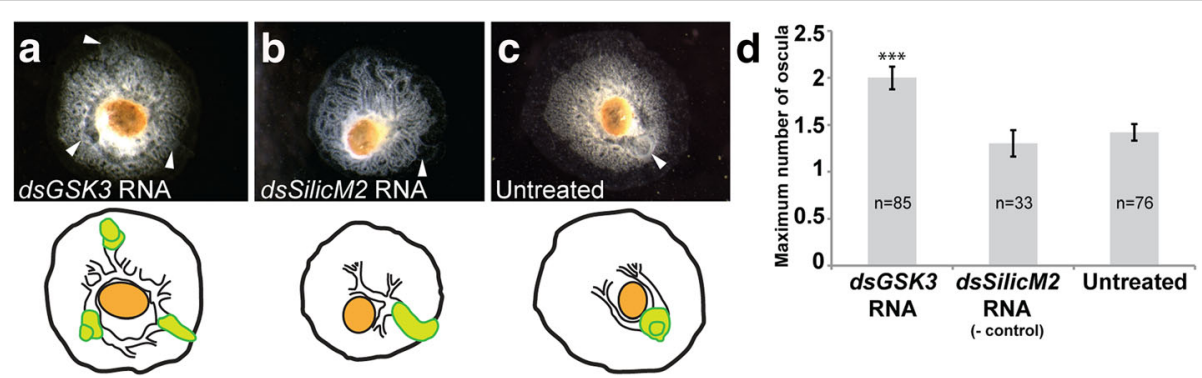

Fig. 4 GSK3 knockdown with dsRNA causes multiple oscula in Ephydatia muelleri. a Treatment with $10 \mu \mathrm{g} / \mathrm{ml}$ GSK3 dsRNA causes multiple oscula (arrowheads) to arise. $\mathbf{b}$ silicatein M2 dsRNA treated sponges (negative control) develop with a single osculum. $\mathbf{c}$ Untreated sponges grown in $1 \times$ M-medium alone typically develop 1 osculum. Lower panel show illustrations to clarify the position of canals in black and oscula in green. $\mathbf{d}$ The mean maximum number of oscula for each treatment. *** indicates $p<0.005$ (Dunn test); difference between controls was not significant $(p>0.05)$. Bars $=$ standard error 
Pharmacological inhibition of Wnt pathway components We treated hatching and regenerating S. lacustris with Wnt pathway inhibitors to test whether they would inhibit the formation of oscula. If inhibition of the negative Wnt signaling regulator GSK-3 causes the formation of ectopic oscula, then inhibiting positive regulators of Wnt signaling should prevent oscular development. Niclosamide has been shown to impede Wnt signaling by causing internalization of the Frizzled receptor, a reduction of dishevelled protein expression, loss of $\beta$-catenin stabilization and a reduction in transcription of Wnt target genes by TCF [28-30]. Park et al. [30] showed that quercetin treatment decreased TCF transcriptional activity, either by acting directly on TCF or on $\beta$-catenin to inhibit their binding, thereby reducing $\mathrm{Wnt}$ target gene expression.

Both niclosamide and quercetin blocked the formation of oscula during hatching and development and both drugs also prevented regeneration of oscular tissue after removal of the osculum (Fig. 5a). Effective concentrations of each drug were determined empirically and narrowed to $0.1-0.2 \mu \mathrm{M}$ niclosamide and 5-10 $\mu \mathrm{M}$ quercetin; at these concentrations oscula were not formed. Control sponges (untreated or treated with $50 \mu \mathrm{M}$ DMSO) developed and regenerated oscula normally (Fig. 5b-j). At high concentrations niclosamide treatment resulted in extreme phenotypes, and often the tissue simply formed a long tube of cells that did not appear to differentiate (Fig. 5d), however at $0.2 \mu \mathrm{M}$ niclosamide sponges lacked oscula and canals but retained a more normal growth pattern (Fig. 5e). When we removed the osculum and treated the sponges with niclosamide, not only did the osculum fail to regenerate, but canal system organization was lost after 48 h (Fig. 5f).

The phenotype of sponges grown in quercetin was less severe than those grown in niclosamide, but at most doses sponges spread out to form mats containing lacunae but no fully-developed, branching canals. Often the sponges formed strands of tissue suggesting growth followed by tissue regression (Fig. 5g). At the highest concentration of quercetin, the osculum did not regenerate and organization of the aquiferous system was lost (Fig. 5h). For comparison, Fig. $5 \mathrm{i}$ and $\mathrm{j}$ show an untreated sponge at $t=0$ and $t=24 \mathrm{~h}$ after osculum removal, respectively. The osculum grew back fully and in the same location (Fig. 5i, inset).

\section{The fate of larval polarity at metamorphosis}

Going into this work our hypothesis was that Wnt signaling was involved in establishing axial polarity and development of the osculum. Our attempts, however, at detecting expression of wht genes in larvae via in situ hybridization were not successful. Therefore, in order to understand whether larval polarity was fixed and retained in the adult or whether tissues were entirely malleable and could reorganize themselves at metamorphosis, we carried out experiments to examine the fate of the poles of the freshwater sponge larvae. Larvae are not produced simultaneously by all colonies of freshwater sponges, and so we used larvae obtained from those individuals and species that were spawning in different years. Following release from adults, swimming larvae of Eunapius fragilis ( $n \geq 100$ each over two years) were bisected perpendicular to their anterior-posterior swimming axis and the halves were cultured separately. Of these, at least half did not settle on coverslips and so could not be followed by microscopy or were lost during processing; here we show the typical results. In E. fragilis anterior halves settled 2030 min after cutting but failed to develop choanocytes and an aquiferous system (Fig. 6b, b'). In contrast, the posterior halves of $E$. fragilis larvae consistently developed multiple oscula with choanocyte chambers and canals, but only $50 \%$ of them settled and metamorphosed (Fig. 6c, c', $\left.\mathrm{d}, \mathrm{d}^{\prime}\right)$. Those that failed to attach continued swimming with oscula - for up to 3 days (Fig. 6d). We did not test whether any of the oscula were functional. The same experiments another year with $S$. lacustris swimming larvae $(n \leq 10)$ gave the opposite results: anterior halves failed to settle and instead continued swimming without further differentiation, while posterior halves settled and grew into normal sponges (Additional file 10). Due to low numbers of available larvae, we were not able to further process or examine bisected larvae from Spongilla lacustris.

We injected the anterior and posterior poles of E. fragilis larvae $(n \geq 100)$ with the fluorescent cell marker diI and tracked the fates of labelled cells through metamorphosis. Only a portion of these were followed through metamorphosis (anterior $n=18$; posterior $n=20$ ) for the same reasons as listed above for bisection experiments. The bulk of dil labelled cells - anterior and posterior typically remained in a diffuse area following larval settlement, but individual cells labelled with diI were found scattered throughout the settler in both cases (Fig. 6e). In both anteriorly and posteriorly labelled larvae, labelled cells differentiated into several different cell types including sclerocytes, choanocytes, archaeocytes and basopinacocytes in the settled sponge (Fig. 6f). However, we only observed cells from the posterior pole becoming cells of the osculum (Fig. $6 \mathrm{~g}$, posterior $n=3 / 20$; anterior $n=0 / 18)$.

\section{Discussion}

Wnt genes and the Wnt signaling pathway were key to the innovations in multicellular metazoan body plan organization [7]. However, several lines of evidence suggest that while individual Wnt pathway components originated in unicells (e.g. [7, 31]), canonical Wnt signaling as we understand it - both mechanistically and for its role in axial polarity - did not arise until after sponges split from the other animals. 


\begin{tabular}{|c|c|c|c|c|}
\hline \multirow[t]{3}{*}{$\mathbf{a}$} & Treatment & $\begin{array}{c}\text { Concentration } \\
(\mu \mathrm{M})\end{array}$ & $\begin{array}{l}\text { Osculum } \\
\text { Growth }\end{array}$ & $\begin{array}{c}\text { Osculum } \\
\text { Regeneration }\end{array}$ \\
\hline & No treatment & - & $100 \%(n=12 / 12)$ & $100 \%(n=6 / 6)$ \\
\hline & \begin{tabular}{|l|} 
DMSO \\
\end{tabular} & 50 & $100 \%(n=36 / 36)$ & $100 \%(\mathrm{n}=40 / 40)$ \\
\hline \multirow{2}{*}{ Frizzled } & \multirow{2}{*}{ Niclosamide } & 0.1 & $58.3 \%(n=7 / 12)$ & $0 \%(n=0 / 12)$ \\
\hline & & 0.2 & $0 \%(n=0 / 12)$ & $0 \%(n=0 / 28)$ \\
\hline \multirow{3}{*}{ Bcat/TCF } & \multirow{3}{*}{ Quercetin } & 1 & $91.7 \%(n=11 / 12)$ & $100 \%(n=12 / 12)$ \\
\hline & & 5 & $25 \%(n=3 / 12)$ & $0 \%(n=0 / 12)$ \\
\hline & & 20 & $0 \%(n=0 / 24)$ & $0 \%(n=0 / 12)$ \\
\hline
\end{tabular}
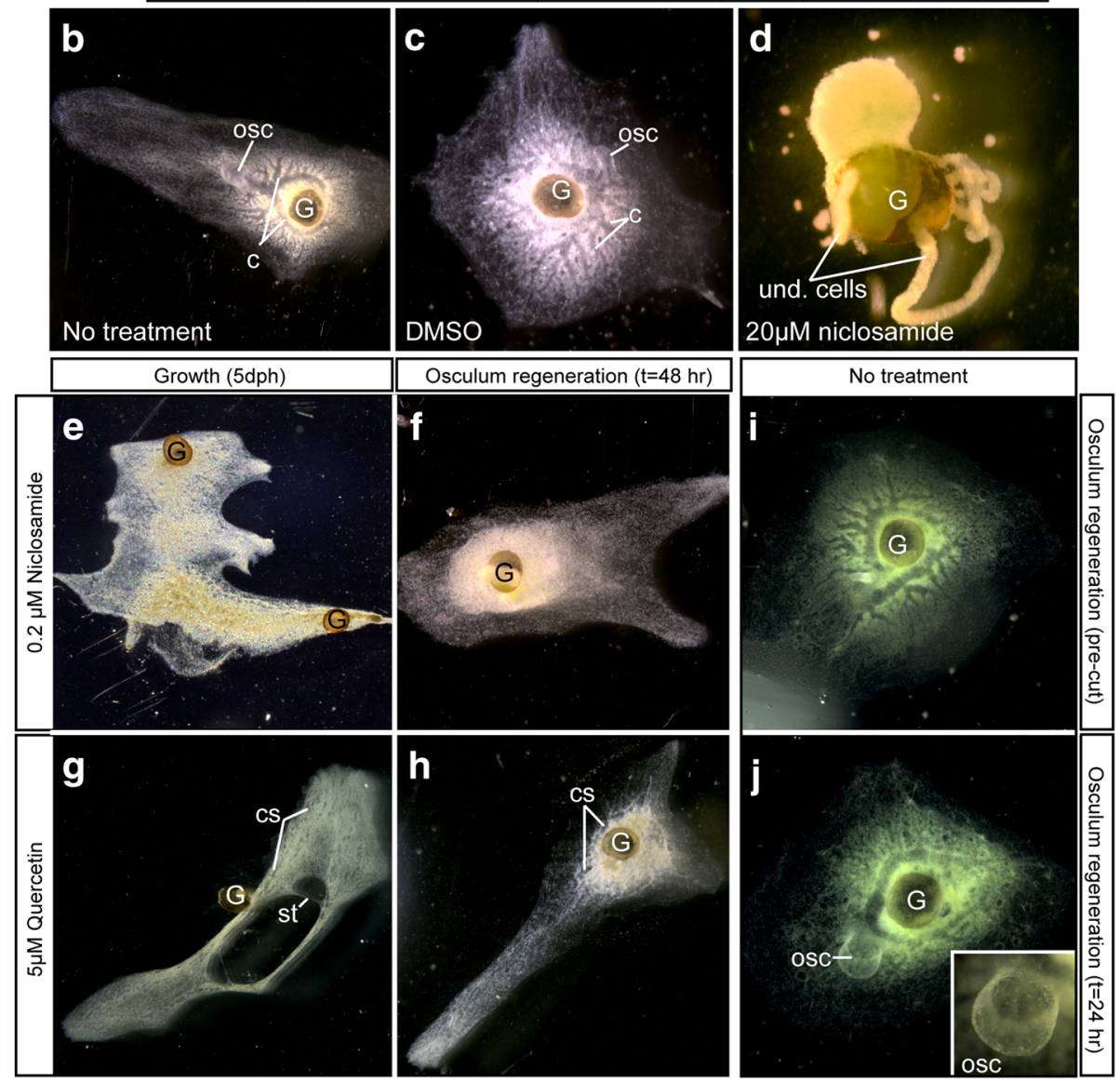

Fig. 5 Pharmacological inhibition of Wnt signaling prevents development and regeneration of oscula in Spongilla lacustris. a) Table showing a summary of treatment results for osculum growth experiments and osculum regeneration experiments. b), c) No treatment and DMSO controls, respectively, showing normal gross morphology of a hatchling, with the gemmule husk (G), an osculum (osc) and canals (c). d) Catastrophic release of undifferentiated cells (und. Cells) after hatching in a high dose of niclosamide ( $20 \mu \mathrm{M})$. e) and $\mathbf{f}$ ) Treatment with $0.2 \mu \mathrm{M}$ niclosamide. e) When gemmules are hatched in niclosamide, oscula and canals do not form by $5 \mathrm{dph}$. $\mathbf{f}$ ) When the osculum is removed from a normal sponge with subsequent niclosamide treatment, sponges fail to regenerate the osculum within $48 \mathrm{~h}$, and also lose canals. g) and $\mathbf{h}$ ) treatment with $5 \mu \mathrm{M}$ quercetin. g) After 5 days of growth, quercetin also prevents osculum development, and canals do not form fully - instead they form canal spaces (cs) that appear distinct from one another in the tissue. These animals also grow and then recede, leaving behind a strand of tissue (st). $\mathbf{h}$ ) Quercetin-treated animals fail to regrow oscula within $48 \mathrm{~h}$ and canals begin to degenerate into canal spaces (cs). i) and $\mathbf{j})$ Osculum regeneration before removal (i) and after $24 \mathrm{~h}$ (j) with no treatment

Wnt pathway components are present in sponges, but wnt genes are conspicuously absent from glass sponges class Hexactinellida, which have uniquely syncytial tissues - and sponge Wnts form subfamilies separate from those of non-sponge animal lineages. Although other work has shown localization of wnt mRNAs to oscula in some sponges, in our study of freshwater sponges, wht expression showed individual cells expressing wht genes throughout the mesohyl of the sponge. Our pharmacological and knockdown experiments are consistent with a role for Wnt signaling in formation of the aquiferous canal system, one identifier of body polarity in a sponge, however our larval manipulation experiments show that conservation of tissue polarity during ontogeny might differ even between sponge genera. Overall the data describe a picture of diverse function of Wnt signaling in sponges. 


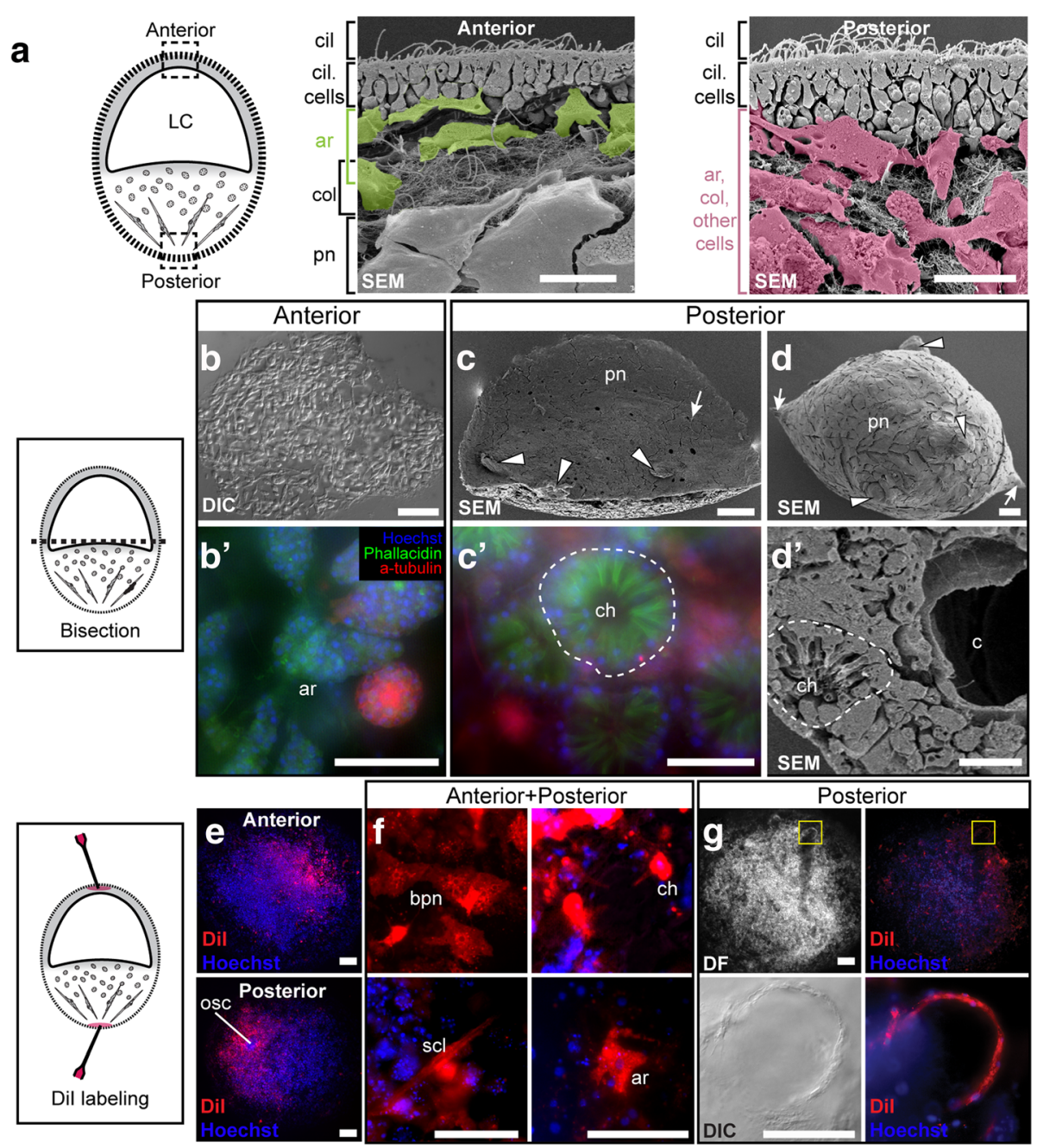

Fig. 6 Bisection and dil labeling of Eunapius fragilis larvae: fate restrictions of anterior and posterior cells. a Body plan of a freshwater sponge larva. The anterior half of the larva is characterized by a larval cavity, and the posterior half contains a mixture of differentiated and undifferentiated cells. Boxed areas, insets: scanning electron micrographs (SEM, scales: $10 \mu \mathrm{m}$ ) of fractures from the anterior half (left) and posterior (right), showing archaeocytes (ar, pseudo-coloured green or pink), collagen (col) and other cells present throughout the larva. The surface of the larva is covered in small ciliated cells $(\sim 2 \times 6 \mu \mathrm{m}$; cells with cilia, cil). The larval cavity is lined by pinacocytes (pn). b A settled anterior hemisphere lacking an osculum, choanocytes and canals. b') These settled anterior halves contained only archaeocytes (ar). c) A fractured settled posterior hemisphere showing a normal pinacoderm (pn) and ostia (arrow) with multiple oscula (arrowheads). c') Normal chaonocyte chambers (ch) in a settled posterior hemisphere. d) Posterior halves also failed to settle in $50 \%$ of cases and remained floating; these had a normal pinacoderm (pn), multiple oscula (arrowheads), and a spicule skeleton (arrows). $\mathbf{d}^{\prime}$ ) Fractured specimen showing canals (c) and choanocyte chambers (ch). $\mathbf{b}^{\prime}$ ) and $\mathbf{c}^{\prime}$ ) nuclei = blue (Hoechst), actin = green (Bodipy fluoresceinphallacidin), tubulin = red (anti-tubulin, Alexa $5942^{\circ}$ antibody). e Overview of labelled cells from anterior or posterior dil tattoos. Cell lineage tracer dye (Dil) is shown in red, nuclei are blue (Hoechst). f) Cells from posterior and anterior poles of larvae are fated to become various cell types including basopinacocytes (bpn), choanocytes (ch), sclerocytes (scl) and archaeocytes (ar). g) Cells at the posterior pole give rise to the osculum (bottom panels show boxed areas; DF = dark field). Scales: b), c), e) and g) $100 \mu \mathrm{m} ; \mathbf{b}^{\prime}$ ) and (') and d) $25 \mu \mathrm{m}$; d') $10 \mu \mathrm{m}$; and f) $50 \mu \mathrm{m}$. Diagrams are modified from versions appearing in [61]

\section{Sponge Wnt relationships}

The presence and absence of Wnts in sponges is an interesting study. We found that three out of four classes of sponges have wnt genes and most of the other major components of the pathway, with major duplications in some groups and probable losses in others.

First, we did not find any Wnts in our transcriptome of Aphrocallistes vastus (Hexactinellida). This result is echoed in a recent study using new transcriptome data from a second hexactinellid, Oopsacas minuta, which also lacks any wnt genes [32]. An interesting probability is that being syncytial, glass sponges may use signaling pathways such as Wnt independently of a ligand; if so, glass sponges could provide a unique model for studying body plan development. This interpretation also suggests that glass sponges may have lost Wnts after they 
diverged from other sponges, and a next important step is to confirm presence/absence of Wnts and Wnt pathway components in other glass sponges by way of a complete genome.

Second, each of the remaining sponge classes has its own complement of Wnts that have diversified within each lineage. Calcareous sponges have more than 20 Wnts [20]. We identified 13 Wnts in the Sycon coactum transcriptome and we were able to assign 11 of these to existing Calcareous sponge Wnts as defined in the above study. The number of Wnts in this class of sponges is surprising only because we tend to associate Wnt signaling with body plan complexity and sponges lack an obviously modular body with complex organs (reviewed in [33]). It is clear from existing data that the Calcarea may have undergone genome duplication events at some time in their evolutionary history causing the appearance of several paralogues of both signaling genes and transcription factors [20, 34]. The function of each of these paralogues or redundancy between them is presently unknown.

Expression and function of Wnt in the freshwater sponge No regional expression of Wnt pathway genes could be detected in Ephydatia muelleri; rather all genes showed a cellular expression pattern. The three $w n t$ genes and $\beta$-catenin are expressed in archaeocytes in the mesohyl at the periphery of the sponge. Some of these archaeocytes appeared to be actively crawling with cytoplasmic extensions reaching out in many different directions. Similar expression was shown for wht in Ephydatia fluviatilis suggesting involvement in spicule organization and arrangement [35], where it was suggested the cells expressing wnt are involved in organizing the positions of spicules. We found no specific co-localization of wnt expression with spicules in our observations. Alternatively, these archaeocytes may be involved in seeking out directions in which the sponge can grow and spread, as this species is an encrusting sponge. Wintermann [36] described two types of crawling archaeocytes, those with and those without filopodia, and these two types respectively express $w n t$ and $\beta$-catenin in Ephydatia muelleri (e.g. [23]).

Based on existing functional and expression work we expected to find wnt expressed in or around the osculum, or perhaps even the ostia [17-22], but we did not find osculum or ostium expression of any wnt or Wnt pathway gene in our sponges. Although it is possible that this is due to a true absence of expression, it is also possible that the tissue of the osculum did not withstand the physical processing of in situ hybridization. Specimens often lost their oscula during the procedure or were heavily damaged despite our best efforts to minimize forces. However, our data is supported by a recent study using a $\beta$-catenin antibody in Ephydatia muelleri that did not reveal staining in the osculum or ostia, but did localize to amoeboid cells as we observed, as well as to some focal adhesion-like structures and substrate-attachment epithelium [23].

Importantly, our work highlights the need to use several controls when applying well-known tools from model systems onto non-models. Our finding that all probes highlight a region adjacent to choanocyte chambers in $5 \mathrm{dph}$ sponges suggests that some cells with inclusions, possibly algal symbionts here, may sequester RNA probes in sponges. Since identification of cell types is difficult in all sponges, and most sponges have cells with inclusions and many also have algal or cyanobacterial symbionts, care must be taken when interpreting regions showing strong labelling in cells with inclusions in sponges. Furthermore, different sponge species have distinct biologies and this should also be taken into account. Clearly a better understanding of sponge cell biology and function will be critical in interpreting how genes work in the sponge.

'Polarity': A role for Wnts in establishment of sponge axis In earlier work we reasoned that the osculum was a definitive marker for sponge 'polarity' [22]. We found that chemicals that activated Wnt signaling in other animals caused the formation of multiple oscula in the freshwater sponge Ephydatia muelleri. We followed this up here using GSK-3 knockdown experiments, and found more oscula formed, as well as pharmacological inhibition of Wnt signaling using quercetin and niclosamide, both of which inhibit Wnt/Frizzled signaling, and found a concentration-dependent inhibition of oscula and aquiferous system development. These results suggest to us that some aspects of the canonical Wnt pathway are involved in formation of the aquiferous system in sponges, but it is not clear to us that this sort of polarity can be properly compared to anterior-posterior polarity in other metazoans.

An important caveat to our pharmacological treatment data is that the actions of these two drugs are not specific to Wnt pathway proteins. Quercetin has many targets including regulators of cell cycle genes and apoptosis, which may explain its role in reducing the growth of cancer cells in rats and cancer cell lines $[37,38]$. Similarly, niclosamide does not exclusively work on Frizzled proteins; it has been shown to inhibit other cell signaling molecules in various types of cancers and other diseases (ERK, Notch, JAKSTAT, NF-k $\beta$ etc.) (Reviewed in [39]). Further studies are required to confirm that the drugs affect the Wnt pathway in sponges.

Sponge larvae have overt anterior-posterior (A-P) swimming polarity [40, 41], and regional expression of some wnt genes along the A-P axis in Sycon ciliatum [20] and at the posterior pole of Amphimedon [18] and the osculum of Halisarca dujardini [21] strongly hints at a role for those Wnts in polarity. However, different wnts are expressed in the posterior pole of the S. ciliatum larva than in the osculum of the juvenile sponge, 
and such a range of expression patterns is seen (in other cells and other regions of the sponge) that it is difficult to know whether polarity is carried over during metamorphosis or re-established de-novo in the juvenile and adult. Our experiments using injection of dye into cells at the posterior and anterior poles of the larva show that in Eunapius fragilis certain elements of larval A-P polarity carries through to the juvenile stage, and more specifically cells at the posterior pole of the larva form the osculum of the juvenile. This is the first confirmation of the conservation of polarity through metamorphosis in a demosponge using cell tracking methods and the finding is significant because the posterior pole of the Amphimedon sponge larvae also expresses wht genes, suggesting that Wnts are involved in determining polarity in sponges as they are in other animals $[17,18,20]$. But we still do not know if gene expression is maintained through metamorphosis. We did not have larvae from Ephydatia muelleri to study and our ISH experiments with larvae from Eunapius fragilis showed no expression, therefore no conclusions regarding the involvement of Wnt signaling and freshwater sponge larval polarity can be drawn.

\section{Methods}

\section{Collection and culture of freshwater sponges}

Adult sponges were collected from lakes near Bamfield, BC for experiments at the Bamfield Marine Sciences Centre and at the University of Alberta. For the RNAi experiment, gemmules were collected in Helena, MT from the site described in [42] and stored at the University of Richmond (Richmond, VA, USA). Specimens at the University of Richmond were imaged on an Olympus SZX12 Stereomicroscope, using ProgRes Camera and the ProgRes Capture software.

Gemmules of S. lacustris and E. muelleri were collected in December and stored at $4{ }^{\circ} \mathrm{C}$ in lake water until use. Gemmules were cleaned of the adult skeleton, treated with hydrogen peroxide and hatched in $1 \times$ M-medium consisting of $0.1 \mathrm{mM} \mathrm{CaCl}, 0.05 \mathrm{mM}$ $\mathrm{MgSO}_{4}, \quad 0.05 \mathrm{mM} \mathrm{NaHCO}, 0.005 \mathrm{mM} \mathrm{KCl}$ and $0.025 \mathrm{mM} \mathrm{Na}_{2} \mathrm{SiO}_{3} \mathrm{pH} 7.0[43,44]$.

Adult specimens of E. fragilis were collected in July 2012 and 2013 from Frederick Lake, near Bamfield, BC. Two to five sponges were kept in $20 \mathrm{~L}$ of lake water at $15-18{ }^{\circ} \mathrm{C}$ and refreshed every 1-2 days. Larvae released from the adult sponge were collected from the surface of the water using a flashlight and a glass Pasteur pipette, and transferred to dishes containing $0.22 \mu \mathrm{m}$ filtered lake water.

General observations of live animals were recorded using a Nikon Coolpix 995 digital camera or a QI-cam mounted on a Zeiss SZX12 Axioskop stereomicroscope. Images were cropped, resized and assembled using Adobe Photoshop and Illustrator CS6.

\section{Transcriptome and phylogenetic analysis}

We used BLAST+ [45] to search our own transcriptomes from 10 species of sponges representing all 4 poriferan classes (from [3] and unpublished data) for Wnt pathway components. The Aphrocallistes vastus, Ephydatia muelleri and Eunapius fragilis transcriptomes were sequenced with a HiSeq2000 at LC Sciences (http://www.lcsciences.com; Houston, TX), while those for Spongilla lacustris, Petrosia ficiformis, Pseudospongosorites suberitoides, Ircinia fasciculata, Chondrilla nucula, Sycon coactum and Corticium candelabrum were sequenced using the Illumina GAII and HiSeq2000 at the Harvard University FAS Center for Systems Biology (Cambridge, MA). Libraries were prepared using the TruSeq RNA sample preparation kit (Illumina, Inc.) and sequences were assembled in CLC Genomics Workbench 5.1 (CLC Bio) (CLC Bio, Aarhaus, Denmark). Other sequence data included in alignments were obtained from online repositories, as listed in Additional file 1.

We constructed phylogenetic trees for both sponge Wnt proteins alone and several metazoan Wnts; sequence accession numbers for phylogenetic analysis are provided in Additional file 11. Only sequences deemed to be full-length or almost full-length were used in phylogenetic analysis. Sequences were aligned using Mafft [46] and trimmed using TrimAL with the 'Automated 1' setting [47]. We constructed 3 types of maximum likelihood trees using PhyML (http://www.atgc-montpellier.fr/phyml/; [48], RAxML Blackbox (http://embnet.vital-it.ch/raxml-bb/ index.php; [49] and IQ-TREE (http://iqtree.cibiv.univie ac.at/; [50]. The final alignment was analyzed using ProtTest 3.4.2 and $\mathrm{LG}+\mathrm{G}+\mathrm{I}$ was determined to be the model that gave the best $\log$ likelihood value $(-\ln L=$ $34,070.97)$ and thus was used in all analyses.

For the PhyML tree, we created a starting BIONJ tree with SPR tree improvement from 5 random starting trees. Support was measured with 1000 bootstrap replicates. This method was considered the most rigorous one. A RAxMLtree was done with 100 bootstrap replicates and the IQ-TREE with 1000 ultrafast bootstrap replicates [51]. A consensus tree was created including bootstrap support values for each analysis. Nodes with support values below 50 were collapsed into polytomies.

\section{Gene expression}

Expression of Wnt pathway genes was studied in 2 and 5 days post hatch (dph) sponges grown from gemmules of Ephydatia muelleri using in situ hybridization. Sponges were grown in $\mathrm{M}$ medium and fixed in $4 \%$ paraformaldehyde (PFA) in $1 / 4$ Holtfreter's solution (HS) $(15 \mathrm{mM} \mathrm{NaCl}$, $0.2 \mathrm{mM} \mathrm{KCl}, 0.2 \mathrm{mM} \mathrm{CaCl}_{2}, 0.6 \mathrm{mM} \mathrm{NaHCO}_{3}$; [52]) with $0.03 \%$ glutaraldehyde overnight at $4{ }^{\circ} \mathrm{C}$. Sponges were rinsed in $1 / 4 \mathrm{HS}$, dehydrated to $100 \%$ ethanol and stored at $-80{ }^{\circ} \mathrm{C}$ until ready to use. Tissue was rehydrated and permeabilized with $5 \mu \mathrm{g} / \mathrm{mL}$ Proteinase $\mathrm{K}$ for $1-2 \mathrm{~min}$, and 
post fixed in 4\% PFA in PBS with $0.1 \%$ Tween-20 before hybridization.

Genes were cloned into the pGEM-T vector (Promega); primers are listed in Additional file 12. Using a PCRgenerated template, reverse transcription was carried out

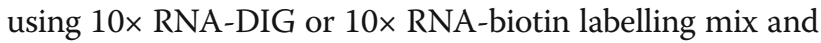
T7 RNA Polymerase (Roche) to generate antisense and sense probes. The products were precipitated with lithium chloride and ethanol and stored at $-20{ }^{\circ} \mathrm{C}$ containing 1 unit RNaseOUT (Invitrogen). Concentration ranges for probes were determined empirically using blots.

Probes were hybridized to sponge tissue for $16-72 \mathrm{~h}$ at $55{ }^{\circ} \mathrm{C}$ in hybridization buffer $(50 \%$ formamide, $5 \times$ SSC, $50 \mu \mathrm{g} / \mathrm{mL}$ heparin salts, $100 \mu \mathrm{g} / \mathrm{mL}$ Torula yeast tRNA, 5× Denhardt's Solution and 0.1\% Tween-20). Post-hybridization washes of $20 \mathrm{~min}$ each were performed at hybridization temperature using post-hybridization solution (50\% formamide, $5 \times$ SSC and $0.1 \%$ Tween-20) in $2 \times \mathrm{SSC}, \mathrm{pH} 4.5$, at a ratio of 3:1, 1:1 and 1:3 respectively, with a final set of $3 \times 20$-min wash in $2 \times \mathrm{SSC} \mathrm{pH} 4.5$. Detection of probes was performed using alkaline phosphatase (AP) conjugated anti-DIG antibody (1:200; Roche), POD conjugated anti-DIG antibody (1:200; Roche), or streptavidin (1:1000; Roche) with Alexa 488 or 594 tyramide reactions. All antibody incubations were performed overnight at $4{ }^{\circ} \mathrm{C}$ on a shaker. Tissue was rinsed in maleic acid buffer $(10 \times 30 \mathrm{~min})$, and colour reactions were performed in the dark at room temperature from $3 \mathrm{~h}$ to overnight depending on probes used. Specimens were labelled with Hoechst 33,342 for nuclei and mounted in Mowiol.

Slides were viewed and imaged on a Zeiss Axioskop 2 Plus compound microscope with a QImaging camera running Northern Eclipse (Empix).

\section{Antibody preparation and labeling}

An antibody to S. lacustris was prepared by injecting rabbits with a fusion protein composed of a intein-chitin tag and residues 1-183 of the S. lacustris $\beta$-catenin protein, which showed $88.4 \%$ sequence identity ( $97.4 \%$ similarity) with the $E$. muelleri $\beta$-catenin protein. The sequence encoding the fusion protein was constructed using the IMPACT kit (New England Biolabs). After expression in Escherichia coli the fusion protein was purified on a chitin column according to the manufacturer's instructions and dialyzed against PBS containing $1 \times$ cOmplete EDTA-free proteinase inhibitor cocktail (Sigma). Peptides were analyzed by mass spectroscopy to confirm sequence identity and injected into rabbits without further processing. The first injection was done in Freunds Complete Adjuvant, and a subsequent three injections in Freunds Incomplete Adjuvant (Sigma).

Serum samples were analyzed by a combination of ELISA and Western blotting. Sponges were fixed and labelled with antibody as described previously [53].

\section{RNA interference}

Double stranded RNA (dsRNA) was generated according to the method used in [25] for EmuGSK-3 and EmuSilicateinM2. Primers are listed in Additional file 11. Gemmules from $E$. muelleri were hatched in $1 \times \mathrm{M}$-medium in 12-well dishes as described above. Shortly after hatching $10 \mu \mathrm{g} / \mathrm{mL}$ dsRNA was added to cultures and fresh solution was exchanged every $24 \mathrm{~h}$ throughout the course of the experiments.

Phenotypes were observed by stereomicroscopy and oscula were scored every $24 \mathrm{~h}$ over 2 days, and confirmed with blind counting of oscula number by a second individual. Imaging was carried out simultaneously. Ostia were not counted as this would require electron microscopy, and inhibition of GSK-3 protein by lithium chloride in this species did not reveal additional ostia [22]. Osculum count data were not normally distributed and had unequal variances, so we used the non-parametric method of Kruskal-Wallis to test for significant differences in the datasets and also confirmed using a Chi Square test. A Dunn test was used to determine difference between treatments [54].

Sponges were stored in RNA Later (Invitrogen) and frozen at $-80{ }^{\circ} \mathrm{C}$. RNA was extracted using the RNeasy kit (Qiagen). Knockdown of GSK-3 was confirmed with qPCR, using EmuEf1- $\alpha$ as a control as described in [25].

\section{Pharmacological experiments}

We treated Spongilla lacustris with niclosamide and quercetin (Sigma-Aldrich) during hatching and growth as well as in osculum regeneration experiments. Each chemical was dissolved in dimethyl sulfoxide (DMSO) to make $10 \mathrm{mM}$ stock solutions. Effective concentrations were determined empirically at a minimum of $0.1 \mu \mathrm{M}$ for niclosamide and $10 \mu \mathrm{M}$ for quercetin in $1 \times \mathrm{M}$-medium. DMSO controls were conducted at up to $50 \mu \mathrm{M}$ in $1 \times$ M-medium.

The presence or absence of an osculum and the number of oscula per dish were recorded for a given number of sponges. In osculum regeneration experiments, sponges were grown in 1× M-medium until oscula developed (5-6 $\mathrm{dph})$. Oscula were manually removed at their bases, and sponges were placed into the respective treatments. Oscula were counted at $7 \mathrm{~h}$ and 1 day post osculum removal.

\section{Larval bisection experiments}

Swimming larvae of Eunapius fragilis were collected before settlement and immobilized by capillary action along the edge of a microscope slide in a Petri dish. Larvae were bisected at their midpoint, perpendicular to the anteriorposterior axis using a $15^{\circ}, 5 \mathrm{~mm}$ restricted depth, straight stab knife (Fine Science Tools) into anterior and posterior hemispheres. These were fixed for electron and fluorescence microscopy immediately or $48 \mathrm{~h}$ after bisection. 
To label anterior and posterior poles of the larva 75$100 \mathrm{~nL}$ of dil prepared at a concentration of $2 \mathrm{mg} / \mathrm{mL}$ in DMSO was injected using an Eppendorf Femtojet into each larva (after [55]). Sponges were cultured to the juvenile stage (24-48 h) and fixed for fluorescence microscopy.

\section{Conclusions}

The cellular expression of wht and Wnt pathway genes in freshwater sponges initially puzzled us, but the diversity of experiments we have carried out now force us to conclude that Wnt signaling does occur in sponges but its role is far more complex than simply designating regionalization of structures. Wnt is likely expressed by cells that act only within a localized region to direct sets of cells for a particular role. Whether those cells will form the osculum, ostia, or pattern canal formation may depend on the lineage of sponge. The absence of Wnts in the glass sponge Aphrocallistes vastus is noteworthy. Glass sponges are syncytial, an unusual tissue construction in which nuclei move through giant syncytia, and in which cellular regions (including archaeocytes) are non-motile and anchored against flagellated chambers. Glass sponges are highly polarized as larvae and as adults and yet if Wnts are truly absent, this polarity is formed and maintained either with a substitute ligand or without Wnt signaling. In either case, it implies that canonical Wnt signaling is not required for polarized larval or adult tissue and body organization in sponges.

A fruitful line of study will be identifying the aspects of sponge body organization that Wnt pathway components are required for, possibly organizing the position of skeletal components, regions of the canal system, or simply choreographing the function of specific sets of cells (e.g., cell adhesion, cell motility). The recent description for a role of the non-canonical Rho-Rock Wnt signaling module in the establishment of the Ephydatia muelleri aquiferous system supports this notion [56]. It is likely that some of these roles are used in other metazoans, reflecting a deep homology of the Wnt signaling pathway. It would be particularly interesting to know whether key interactions between fundamental components of the Wnt pathway pre-date and formed the first steps in metazoan evolution.

\section{Additional files}

Additional file 1: List of sources of sequence data obtained for phylogenetic analysis. (PDF $81 \mathrm{~kb}$ )

Additional file 2: Mafft alignment of sponge Wnt protein sequences prepared in Boxshade (http://www.ch.embnet.org/software/ BOX_form.html). Conserved cysteine residues are marked with a red asterisk, and the conserved RWNC motif is indicated with a green bracket. (PDF $9686 \mathrm{~kb}$ )

Additional file 3: Raw phylogenetic trees used to create the consensus tree presented in Fig. 1. A) PhyML tree with support values from 1000 bootstrap replicates. B) RAxML tree showing bootstrap support from 100 replicates. C) IQ-TREE with support values from $1000 \mathrm{SH}$-aLRT replicates/ aBayes/1000 ultrafast bootstrap replicates. (PDF $820 \mathrm{~kb}$ )

Additional file 4: Assignment of sponge Wnts to each sponge-specific Wnt subfamily. Groupings were decided by several phylogenetic analyses and are taken from the consensus tree shown in Fig. 1, and subfamily names were retained from [18-20]. (PDF $65 \mathrm{~kb}$ )

Additional file 5: Raw phylogenetic PhyML tree with sponge and bilaterian Wnt sequences. Values displayed are bootstrap support from 100 replicates. Species codes: Nve = Nematostella vectensis, Dre = Danio rerio, $\mathrm{Hsa}=$ Homo sapiens, sponge species codes are as listed in the legend of Fig. 1. (PDF 585 kb)

Additional file 6: Western blot validation of our custom antibody to a $\beta$-catenin fusion protein from Spongilla lacustris. (PDF $349 \mathrm{~kb}$ )

Additional file 7: Fluorescent in situ hybridization and antibody images showing separate channels for images shown in Fig. 2. A) wntB, B) wntA/ wntC, C) wntA/wntB and D) $\beta$-catenin antibody. (TIFF $6439 \mathrm{~kb}$ )

Additional file 8: In situ hybridization control experiments. A) A dual probe from Danio rerio against hemoglobin $(h b)$ and engrailed (en) shows a brightly labelled region next to choanocyte chambers (arrowhead). B) Dual probe from $D$. rerio co-labelled with a dsh probe, showing the same regions labelled next to chaonocyte chambers (arrowhead). Co-staining indicates a lack of full specificity of the sponge $d$ sh probe. C) Low magnification view of a sense probe control for silicatein M2 (silcM2). The boxed area is shown to the right at higher magnification showing diffuse, low-level staining of many cells and structures, including spicules. D) Fluorescent silcM2 label for comparison showing brightly labelled sclerocytes surrounding spicules, and no background staining. Boxed area is shown to the right at a higher magnification. E) Nuclei labelled with hoechst (left), tissue autofluorescence when no probe is applied (middle), and a DIC overview of the region pictured (right). Scales $=50 \mu \mathrm{m}$. (TIFF $7044 \mathrm{~kb})$

Additional file 9: Confirmation of gsk3 knockdown by qPCR. Relative expression levels of gsk3 in untreated versus dsRNA treated sponges (dsRNA EmuGSK3). Expression levels normalized to Ef1-a. (TIFF 319 kb)

Additional file 10: Bisection experiments in Spongilla lacustris larvae have the opposite result of that seen in E. fragilis. Larval appearance is very similar to E. fragilis. However, in S. lacustris the posterior hemisphere settles and forms a normal sponge while the anterior half remains undifferentiated and continues swimming in the water column for up to 3 days. (TIFF $2871 \mathrm{~kb}$ )

Additional file 11: Accession numbers of previously published sequences used for phylogenetic analysis in Fig.1 and Additional files 3 and 5. (PDF $64 \mathrm{~kb}$ )

Additional file 12: Primers used to amplify sequences for RNAi and mRNA injection experiments. (PDF $66 \mathrm{~kb}$ )

\section{Abbreviations}

Aar: Aardvark; AP: Alkaline phosphatase; A-P: Anterior posterior; BIO: 6bromoindirubin-3'-oxime; BLAST: Basic Local Alignment Search Tool; DIG: Digoxigenin; dil: 3H-Indolium,2-[3-(1,3-dihydro-3,3-dimethyl-1octadecyl-2H-indol-2-ylidene)-1-propenyl]-3,3-dimethyl-1-octadecyl,perchlorate; dkk: dickkopf; DMSO: Dimethylsulfoxide; dph: days post hatch; dsh: dishevelled; dsRNA: double-stranded RNA; Ef1a: Elongation factor 1-alpha; ERK: Extracellular-signal regulated kinase; FISH: Fluorescent in situ hybridization; Fz: Frizzled; GSK-3: Glycogen synthase kinase 3; HS: Holtfreter's Solution; JAK-STAT: Janus Kinase-Signal Transducer and Activator of Transcription; mRNA: messenger RNA; NBT/BCIP: Nitroblue tetrazolium salts/5-bromo-4-chloro-3-indolyl-phosphate; NF-kß: Nuclear factor kappa-light-chain-enhancer of activated B cells; PBS: Phosphate buffered saline; PCR: Polymerase chain reaction; PFA: Paraformaldehyde; POD: Peroxidase; qPCR: quantitative PCR; RNA: Ribonucleic acid; RNA: RNA sequencing; RNAi: RNA interference; SSC: Saline-sodium citrate; TCF-LEF: Transcription factor/lymphoid enhancing-binding factor proteins; tRNA: transfer RNA; Wnt: Wingless/integrated superfamily proteins; $\beta$ cat: $\beta$-catenin 


\section{Acknowledgements}

We thank the director and staff at Bamfield Marine Sciences Centre, J. Mah and J. Weekes for help with larval experiments, and G. Thomsen and W. Gillis (Stony Brook University) for their support with parts of this work. We acknowledge T. Locke and A. Cornish (Molecular Biology Service Unit) and A. Oatway (Advanced Microscopy Facility) for technical assistance, A. Kahn and R. Brown for advice on statistics and D. Pilgrim, W. Gallin for critical evaluations of this work.

\section{Funding}

This work was supported by an NSERC Discovery Grant to SPL, NSERC PGS-D to PWR, an NSF Award (grant number 0829763) to ALH, and an HHMI Grant (grant number 52007567) to DP.

\section{Availability of data and materials}

The transcriptome datasets for Ephydatia muelleri and Eunapius fragilis and other data used and analyzed in the current study are available on the University of Alberta Education and Research Archive (ERA; Aphrocallistes vastus: https://doi.org/10.7939/R35000 [57]; Ephydatia muelleri: https:/doi.org/ 10.7939/R3WH2DV20 [58]; Eunapius fragilis: https://doi.org/10.7939/R3794177K [59]. Relevant phylogenetic data has also been uploaded to the ERA at https://doi.org/10.7939/R3PN8XV7D [60].

\section{Authors' contributions}

PWR, SPL, DP and ALH conceived and designed the study, PWR performed larval experiments, phylogenetics, in situ hybridizations and antibody production and labelling, PWR and DP performed RNAi experiments and PWR, EM and AM performed the pharmacological inhibitions. PWR, ALH and SPL wrote the manuscript. All authors read and approved the final manuscript.

\section{Ethics approval and consent to participate}

All protocols were performed according to the guidelines stated by the Canadian Council for Animal Care and the protocols approved by the Animal Care and Use Committee of the University of Alberta (Animal Use Number: AUP00000840)

\section{Consent for publication}

Not applicable.

\section{Competing interests}

The authors declare that they have no competing interests.

\section{Publisher's Note}

Springer Nature remains neutral with regard to jurisdictional claims in published maps and institutional affiliations.

\section{Author details}

'Department of Biological Sciences, University of Alberta, Edmonton, AB, Canada. ${ }^{2}$ Department of Biological Sciences, MacEwan University, Edmonton, $A B$, Canada. ${ }^{3}$ Department of Biology, University of Richmond, Richmond, VA, USA. ${ }^{4}$ Department of Molecular Genetics and Microbiology, Duke University, Durham, NC, USA.

\section{Received: 9 August 2017 Accepted: 4 January 2018}

\section{Published online: 02 February 2018}

\section{References}

1. Larroux C, Luke GN, Koopman P, Rokhsar DS, Shimeld SM, Degnan BM. Genesis and expansion of metazoan transcription factor gene classes. Mol Biol Evol. 2008;25(5):980-96.

2. Richter DJ, King N. The genomic and cellular foundations of animal origins. Annu Rev Genet. 2013:47:509-37.

3. Riesgo A, Farrar N, Windsor PJ, Giribet G, Leys SP. The analysis of eight transcriptomes from all poriferan classes reveals surprising genetic complexity in sponges. Mol Biol Evol. 2014;31(5):1102-20.

4. King N, Westbrook MJ, Young SL, Kuo A, Abedin M, Chapman J, Fairclough $\mathrm{S}$, Hellsten U, Isogai Y, Letunic I, et al. The genome of the choanoflagellate Monosiga brevicollis and the origin of metazoans. Nature. 2008:451(7180): 783-8.
5. Sebe-Pedros A, de Mendoza A, Lang BF, Degnan BM, Ruiz-Trillo I. Unexpected repertoire of metazoan transcription factors in the unicellular holozoan Capsaspora owczarzaki. Mol Biol Evol. 2011;28(3):1241-54.

6. Fairclough S, Chen Z, Kramer E, Zeng Q, Young S, Robertson HM, Begovic E, Richter DJ, Russ C, Westbrook MJ, et al. Premetazoan genome evolution and the regulation of cell differentiation in the choanoflagellate Salpingoeca rosetta. Genome Biol. 2013;14:R15.

7. Loh KM, van Amerongen R, Nusse R. Generating cellular diversity and spatial form: Wnt signaling and the evolution of multicellular animals. Dev Cell. 2016;38(6):643-55.

8. Kusserow A, Pang K, Sturm C, Hrouda M, Lentfer J, Schmidt HA, Technau U, von Haeseler A, Hobmayer B, Martindale MQ, et al. Unexpected complexity of the Wnt gene family in a sea anemone. Nature. 2005;433:156-60.

9. Hassel $M$, Albert $K$, Hofheinz $S$. Pattern formation in Hydra vulgaris is controlled by lithium-sensitive processes. Dev Biol. 1993;156:362-71.

10. Wikramanayake A, Hong M, Lee PN, Pang K, Byrum CA, Bince JM, Xu R, Martindale MQ. An ancient role for nuclear $\beta$-catenin in the evolution of axial polarity and germ layer segregation. Nature. 2003;426:446-50.

11. Guder C, Pinho S, Nacak TG, Schmidt HA, Hobmayer B, Niehrs C, Holstein TW. An ancient Wnt-Dickkopf antagonism in hydra. Development. 2006;133(5):901-11.

12. Philipp I, Aufschnaiter R, Ozbek S, Pontasch S, Jenewein M, Watanabe $H$, Rentzsch F, Holstein TW, Hobmayer B. Wnt/beta-catenin and noncanonical Wnt signaling interact in tissue evagination in the simple eumetazoan hydra. P Natl Acad Sci USA. 2009;106(11):4290-5.

13. Kraus Y, Fritzenwanker JH, Genikhovich G, Technau U. The blastoporal organiser of a sea anemone. Curr Biol. 2007;17(20):R874-6.

14. Harwood AJ. Dictyostelium development: a prototypic Wnt pathway? In: Vincan E, editor. Wht Signalling volume II: pathway models. New York, NY: Springer + Business Media; 2008. p. 21-32.

15. Jager M, Dayraud C, Mialot A, Queinnec E, le Guyader H, Manuel M. Evidence for involvement of Wht signalling in body polarities, cell proliferation, and the neuro-sensory system in an adult ctenophore. PLoS One. 2013;8(12):e84363.

16. Pang K, Ryan JF, Program NCS, Mullikin JC, Baxevanis AD, Martindale MQ. Genomic insights into Wnt signaling in an early diverging metazoan, the ctenophore Mnemiopsis leidyi. EvoDevo. 2010;1(1):10.

17. Adamska M, Degnan SM, Green KM, Adamski M, Craigie A, Larroux C, Degnan BM. Wnt and TGF-beta expression in the sponge Amphimedon queenslandica and the origin of metazoan embryonic patterning. PLoS One. 2007;2(10):e1031.

18. Adamska M, Larroux C, Adamski M, Green K, Lovas E, Koop D, Richards GS, Zwafink C, Degnan BM. Structure and expression of conserved Wnt pathway components in the demosponge Amphimedon queenslandica. Evol Dev. 2010;12(5):494-518.

19. Lapebie P, Gazave E, Ereskovsky A, Derelle R, Bezac C, Renard E, Houliston E, Borchiellini C. WNT/beta-catenin signalling and epithelial patterning in the homoscleromorph sponge Oscarella. PLoS One. 2009;4(6)::5823.

20. Leininger S, Adamski M, Bergum B, Guder C, Liu J, Laplante M, Brate J, Hoffmann F, Fortunato S, Jordal S, et al. Developmental gene expression provides clues to relationships between sponge and eumetazoan body plans. Nat Commun. 2014;5:3905.

21. Borisenko I, Adamski M, Ereskovsky A, Adamska M. Surprisingly rich repertoire of Wnt genes in the demosponge Halisarca dujardini. BMC Evol Biol. 2016;16(1):123.

22. Windsor PJ, Leys SP. Wnt signaling and induction in the sponge aquiferous system: evidence for an ancient origin of the organizer. Evol Dev. 2010;12(5): 484-93.

23. Schippers KJ, Nichols SA. $\beta$-catenin has both conserved and novel functions in the sponge Ephydatia muelleri. BioRxiv. 2017; https:/doi.org/10.1 101/164012.

24. Mohri K, Nakatsukasa M, Masuda Y, Agata K, Funayama N. Toward understanding the morphogenesis of siliceous spicules in freshwater sponge: differential mRNA expression of spicule-type-specific silicatein genes in Ephydatia fluviatilis. Dev Dyn. 2008;237(10):3024-39.

25. Rivera AS, Hammel JU, Haen KM, Danka ES, Cieniewicz B, Winters IP, Posfai D, Worheide G, Lavrov DV, Knight SW, et al. RNA interference in marine and freshwater sponges: actin knockdown in Tethya wilhelma and Ephydatia muelleri by ingested dsRNA expressing bacteria. BMC Biotechnol. 2011;11:67.

26. Rivera A, Winters I, Rued A, Ding S, Posfai D, Cieniewicz B, Cameron K, Gentile $L$, Hill A. The evolution and function of the Pax/six regulatory network in sponges. Evol Dev. 2013;15(3):186-96. 
27. Cramer JM, Pohlmann D, Gomez F, Mark L, Kornegay B, Hall C, Siraliev-Perez E, Walavalkar NM, Sperlazza MJ, Bilinovich S, et al. Methylation specific targeting of a chromatin remodeling complex from sponges to humans. Sci Rep. 2017;7:40674.

28. Chen M, Wang J, Lu J, Bond MC, Ren XR, Lyerly HK, Barak LS, Chen W. The anti-helminthic niclosamide inhibits Wnt/Frizzled1 signaling. Biochemistry. 2009;48(43):10267-74.

29. Chen W, Chen M, Barak LS. Development of small molecules targeting the Wnt pathway for the treatment of colon cancer: a high-throughput screening approach. Am J Physiol-Gastr L. 2010;299(2):G293-300.

30. Park CH, Chang JY, Hahm ER, Park S, Kim HK, Yang CH. Quercetin, a potent inhibitor against beta-catenin/Tcf signaling in SW480 colon cancer cells. Biochem Biophys Res Co. 2005;328(1):227-34.

31. Holstein TW. The evolution of the Wnt pathway. CSH Perspect Biol. 2012:4(7):a007922.

32. Schenkelaars Q, Pratlong M, Kodjabachian L, Fierro-Constain L, Vacelet J, Le Bivic A, Renard E, Borchiellini C. Animal multicellularity and polarity without Wnt signaling. Sci Rep. 2017;7:15383.

33. Leys SP, Hill A. The physiology and molecular biology of sponge tissues. Adv Mar Biol. 2012;62:1-56.

34. Fortunato SA, Adamski M, Ramos OM, Leininger S, Liu J, Ferrier DE, Adamska M. Calcisponges have a ParaHox gene and dynamic expression of dispersed NK homeobox genes. Nature. 2014;514(7524):620-3.

35. Manuel M, Foret S. Searching for eve: basal metazoans and the evolution of multicellular complexity. BioEssays. 2012;34(3):247-51.

36. Wintermann G. Entwicklungsphysiologische Untersuchungen an Süßwasserschwämmen. Zool Jahrb Allg Zool. 1950;71:427-86.

37. Matsukawa $Y$, Nishino H, Okuyama $Y$, Matsui T, Matsumoto T, Matsumura S, Shimizu Y, Sowa Y, Sakai T. Effects of quercetin and/or restraint stress on formation of aberrant crypt foci induced by Azoxymethane in rat colons. Oncology. 1997;54(2):118-21.

38. Wenzel U, Herzog A, Kuntz S, Daniel H. Protein expression profiling identifies molecular targets of quercetin as a major dietary flavonoid in human colon cancer cells. Proteomics. 2004:4(7):2160-74.

39. Chen W, Mook RA, Premont RT, Wang J. Niclosamide: beyond an antihelminthic drug. Cell Signal. 2017; https://doi.org/10.1016/j.cellsig.2017.04.001.

40. Leys SP, Degnan BM. Cytological basis of photoresponsive behavior in a sponge larva. Biol Bull. 2001;201(3):323-38.

41. Maldonado M, Bergquist P. Phylum Porifera. In: Young CM, editor. Atlas of marine invertebrate larvae. San Diego: academic press; 2003. p. 21-50.

42. Peterson KJ, Addis JS. Clypeatula cooperensis gen. N., sp. n., a new freshwater sponge (Porifera, Spongillidae) from the Rocky Mountains of Montana, USA. Zool Scr. 2000;29(3):265-374.

43. Elliott GR, Leys SP. Coordinated contractions effectively expel water from the aquiferous system of a freshwater sponge. J Exp Biol. 2007;210(Pt 21): 3736-48.

44. Rasmont R. Une technique de culture des éponges d'eau douce en milieu controlé. Ann Soc Roy Zool Bel. 1961;91:147-56.

45. Camacho C, Coulouris G, Avagyan V, Ma N, Papadopoulos J, Bealer K, Madden TL. BLAST+: architecture and applications. BMC Bioinformatics. 2009;10:421.

46. Katoh K, Standley DM, Multiple Sequence MAFFT. Alignment software version 7: improvements in performance and usability. Mol Biol Evol. 2013;30(4):772-80

47. Capella-Gutiérrez S, Silla-Martínez JM, Gabaldón T. trimAl: a tool for automated alignment trimming in large-scale phylogenetic analyses. Bioinformatics. 2009;25(15):1972-3.

48. Guindon S, Dufayard J-F, Lefort V, Anisimova M, Hordijk W, Gascuel O. New algorithms and methods to estimate maximum-likelihood phylogenies: assessing the performance of PhyML 3.0. Syst Biol. 2010;59(3):307-21.

49. Stamatakis A, Hoover P, Rougemont J, Renner S, Rapid Bootstrap A. Algorithm for the RAxML web servers. Syst Biol. 2008;57(5):758-71.

50. Nguyen L-T, Schmidt HA, von Haeseler A, Minh BQ. IQ-TREE: a fast and effective stochastic algorithm for estimating maximum-likelihood phylogenies. Mol Biol Evol. 2015;32(1):268-74.

51. Minh BQ, Nguyen MAT, von Haeseler A. Ultrafast approximation for phylogenetic bootstrap. Mol Biol Evol. 2013;30(5):1188-95.

52. Spiegel M. The reaggregation of dissociated sponge cells. Ann N Y Acad Sci. 1955;60(7):1056-78.
53. Ludeman DA, Farrar N, Riesgo A, Paps J, Leys SP. Evolutionary origins of sensation in metazoans: functional evidence for a new sensory organ in sponges. BMC Evol Biol. 2014;14:3.

54. Dunn OJ. Multiple comparisons using rank sums. Technometrics. 1964;6(3): 241-52.

55. Adamska M, Degnan BM. Analysis of cell movement in Amphimedon embryos by injection of fluorescent tracers. CSH Protocols. 2008;3(12). https://doi.org/10.1101/pdb.prot5097.

56. Schenkelaars Q, Fierro-Constain L, Renard E, Hill AL, Borchiellini C. Insights into frizzled evolution and new perspectives. Evol Dev. 2015;17(2):160-9.

57. Leys SP. Aphrocallistes vastus trinity transcriptome. University of Alberta Education and Research Archive. 2014; https://doi.org/10.7939/R3S000.

58. Leys SP. Ephydatia muelleri trinity transcriptome. University of Alberta Education and Research Archive. 2017; https://doi.org/10.7939/R3WH2DV20.

59. Leys SP. Eunapius fragilis trinity transcriptome. University of Alberta Education and Research Archive. 2017; https://doi.org/10.7939/R3794177K.

60. Leys SP, Windsor Reid PJ, Hill A. Phylogenetic analyses and fasta files of Porifera Wnts. University of Alberta Education and Research Archive. 2017; https://doi.org/10.7939/R3PN8XV7D.

61. Windsor PJ. Evolution of the sponge body plan: Wnt and the development of polarity in freshwater sponges. Thesis: University of Alberta; 2014

\section{Submit your next manuscript to BioMed Central and we will help you at every step:}

- We accept pre-submission inquiries

- Our selector tool helps you to find the most relevant journal

- We provide round the clock customer support

- Convenient online submission

- Thorough peer review

- Inclusion in PubMed and all major indexing services

- Maximum visibility for your research

Submit your manuscript at www.biomedcentral.com/submit 